\title{
Mi experiencia docente en el contexto de las aulas costarricenses: el disfrute de retomar las acciones del pedagogo, inspiraciones en el Repertorio Americano
}

Saray Elena Loáiciga Brenes

Sede Chorotega, Universidad Nacional Ana Lorena Camacho Camacho Sede Guanacaste, Universidad de Costa Rica

\section{Resumen}

En nuestra propia práctica docente, nos encontramos con una gran diversidad del alumnado que tiene historias de vida, formas de crianza diferente, ritmo y estilos de aprendizaje propios, que nos hacen meditar hacia dónde enrumbar nuestros esfuerzos académicos y de formación integral; ayudarles es la satisfacción más maravillosa por la cual pasamos los profesores. Son cientos de rostros humanos que hacen posible la tarea de educar. Y detrás de un educador hay personas inspiradoras que contribuyeron a confirmar la orientación para el arte de educar. Por ello, este trabajo hace un recorrido por varias experiencias gratificantes de los educadores como artistas que pintan el porvenir de muchos para que sean los baluartes de una sociedad solidaria.

Palabras claves: educar, inspiración, escenarios educativos, prácticas docentes 


\begin{abstract}
During our own practice, we find a great diversity of students who have life stories, different ways of breeding, rhythm and styles of their own learning, which make us inspire our academic and comprehensive training efforts; to help them is the most wonderful satisfaction and why we teach. There are hundreds of human faces that make the task of educating. And behind them an inspiring educator who helped to confirm the orientation for the art of education. Therefore, this work makes a tour of several rewarding experiences of educators and artists who paint the future of many to be the bulwarks of a caring society.
\end{abstract}

Keywords: education, inspiration, educational settings, teaching practices

$\Lambda$ demás, la obra del avance humano hacia una saludable redención no puede hacerse de un golpe, por un acuerdo espontáneo de todos los hombres: es la obra de pequeños grupos, de la propaganda tenaz por largos años y dentro de los límites familiares del país, de la ciudad, de la aldea en que nacimos. El amor a la humanidad, en el amplio sentido de la prédica, de puro extenso se diluye y no pasa de una amable intención. (Repertorio Americano, 30 de abril, 1923)

Por nuestras venas corren sensaciones de regocijo, de ternura, de orgullo, de placer al darnos cuenta por ellos de que fuimos alguien importante en sus vidas. Lastimosamente no aprendimos a sistematizar todas nuestras vivencias, porque si hubiese sido así posiblemente en este momento tendríamos que publicar muchos libros de todas nuestras prácticas pedagógicas. Pues no sé si a ustedes les pasa, pero cuando tengo tiempo de pensar por dónde he transitado, me llena saber que hemos tocado la vida de muchas personas; es tan excitante pensar en los bellos recuerdos que poseemos y decir ¡cómo ha pasado el tiempo! En cada recuerdo y acto educativo realizado, se cristalizan nuestras obras como formadores.
Las personas que buscan lo que les apasiona, contribuyen a la unión de todo el universo; se dice que la unidad del mundo depende de la búsqueda de lo que nos apasiona. La vida surge en respuesta al encanto que poseemos internamente y deseamos exteriorizarlo hacia otros; esta secuencia revela el sentido del esfuerzo humano.

Enseñar lo mágico, lo absurdo, lo misterioso es hacer viajes insospechados por los caminos del aprendizaje. Descubrir y descubrirse a uno mismo permite el deleite y la transformación con la plena seguridad de que nuestra conciencia se libera y nuestra mente se abre a un mundo de posibilidades.

La mediación pedagógica busca que los procesos tengan aprendizaje con experiencias significativas, llenas de gozo, placer, novedosas; por ello, la expresividad es un talento que debe desarrollarse mediante espacios donde se opine, discuta, critique con claridad, con fundamento, coherencia, seguridad. Promoverla en sus tres dimensiones: verbal, oral y escrita. Respetar las opiniones e ideas ajenas es permitir que cada persona se sienta protagonista. Estas experiencias deben lograr la interlocución y la interactividad entre los aprendientes. 
Abro la puerta... de mi vida y ahí están presentes los rostros de María, Ramón, Sofía, Pedro, Cristina, Juan, Matilde, Geovanny, Juana, Óscar, Auxiliadora, Fabián, Vilma, Rosa, Julián, Federico, Karina, Johnny, Michelle, Vanessa, Fernando, Alejandra, William, Carolina, José Mario, Bryan, María, Dimas, Marlon, Karla, Javier, Virya, Miguel, Orlando, Erick, Grace, Mauricio, Víctor, Elieth, Diego, Karol ...y un sinfín de niños, niñas, jóvenes, quienes han transitado por la educación y he tenido el honor de compartir con ellos... Llantos, risas, quejas, juegos, pleitos, sonrisas, tristezas, temores salen a flote en cada uno de ellos, sedientos de ser escuchados y atendidos por una mano amiga. Cuando ellos y ellas se van para sus casas, los edificios educativos pierden fuerza, pierden vida y se sumergen en un estado de desolación, agonía, en espera de un nuevo día de clases; sin ellos la escuela desfallece lentamente, todo pierde sentido. Son las almas vivas que vivifican las instituciones.

Miles de miradas sueñan con llegar a la escuela, y ser felices, en un espacio donde una vez que ponen los pies sobre la tierra, se someten a una serie de aventuras que les permiten satisfacer sus necesidades de solidaridad, amistad, experiencias, conocimientos. Los vemos llegar, pero de igual forma irse. Dejan atrás una serie de vivencias que les marcarán la vida para siempre. Y nos marcan a nosotros la vida, nos hacen sentir importantes en su formación, somos el refugio, la confianza, la amistad, el respeto y la mamá amorosa, pero firme, que les corrige, les ayuda, les explica cuando no entienden. Pero de igual forma los maestros constituyen en muchos casos la figura masculina, que representa a ese papá que muchos no tienen y suplen así una necesidad afectiva. Mirarlos en sus necesidades es la posibilidad de encontrarnos frente a frente para compartir esas angustias, temores, tristezas y ayuda que piden a gritos de una persona que está a su lado, inspiradora de confianza y apoyo para lo que necesiten.

No es nada fácil encontrarnos con una gran diversidad del alumnado que tiene sus propias historias de vida. Son miles de anécdotas que las guardamos en nuestro baúl de los recuerdos. Cómo olvidar a Alexander, un niño de 8 años, que ingresó en 1992 a segundo año a la escuela, provenía de Nicaragua. Llegó a la puerta y dijo a viva voz: ¡muy buenos días!, ¿cómo amanecieron mis hermanos y hermanas? -Yo bendecido y agradecido con Dios, espero que ustedes también. Entró al aula, se sentó y se acomodó. Su mirada era profunda, ojitos negros, una sonrisa tierna, su carita estaba gozosa de alegría, se le veía reír y satisfacerse por estar ahí. No le importó que muchas miradas vieran sus zapatos viejos pero limpios; una camisa blanca casi amarilla, posiblemente el exceso de cloro la puso así; su bolsita plástica la acomodó dentro de la mesa, ahí llevaba sus cuadernos y el único pedazo de lápiz que le acompañaba. Y de manera respetuosa y atenta se dispuso a escuchar a su maestra. Ese niño cargaba, ese primer día de clase, todas las ilusiones del mundo, una alegría que se desprendía de él, la fe y la esperanza de recibir la mejor educación del mundo de parte de la maestra, sabía de antemano que ella era la encargada de hacer de su estadía en la escuela los momentos más significativos de su aprendizaje. Ese niño desprendió todo aquel sentido de por qué se 
educa. Y máxime cuando vemos que viene de otra cultura y educación que trae incorporadas, que lo hacen a él muy valiente porque tiene que adaptarse a otra cultura y educación diferentes a las de su país. Leía cancaneado, con un fuerte acento producto del método fonético, pero su esfuerzo y dedicación sobrepasaron cualquier dificultad de adaptación y asimilación. Él venció cualquier barrera idiomática y cultural para entregarse plenamente a una formación educativa para su vida.

Es ese ser humano el que inspira todo accionar educativo, ¿quién de nosotros no estaría motivado de la actitud de ese niño, dispuesto a aprender a cualquier costo? Hoy tiene 21 años, me lo encontré un día de estos en la calle, me saludó efusivamente, con una mirada tan tierna que me conmovió de inmediato, me abrazó fuertemente y me dijo: Niña, ¡cómo que no iba a acordarme de usted, que me enseñó mucho y hoy estoy estudiando una carrera! Ese momento mágico trascendió mis sentidos, me cobijó una alegría interna sabrosa de esas que no están tan frecuentes en nuestras vidas. Después de conversar largo y tendido, se despidió con una mirada de nostalgia: Ay niña, fue un gusto verla, de verdad que sí, te quiero mucho. Y se fue lentamente viendo de vez en cuando hacia atrás. Yo me quedé viéndole hasta que dobló en una esquina y se me perdió.

Ese día iba a cruzar la calle, miraba hacia mi izquierda para observar en qué momento iba a tener oportunidad de pasar la calle. Estaba tan concentrada en que no pasara ningún carro, cuando iba a hacer el intento para pasarme a la otra acera, alguien me sujetó por los hombros. En segundos la vida se me fue, estaba en la espera cuando sentí a mis espaldas que una persona muy alta me tomó en sus brazos y me apretó, para decirme tiernamente: ¡Mi linda maestra, hasta que por fin te veo! Me volví despacito para ver la cara de ese extraño que me hablaba, cuando lo miré fue acordarme unos veintidós años atrás, cuando tuve la oportunidad de tenerlo en mi clase, era muy fogoso, siempre andaba buscando la forma de distraer a los demás chiquillos, se levantaba sin cesar, se reía de sus fechorías, él era único, no había otro igual.

Al finalizar las clases, eso sí, se quedaba conmigo, me ayudaba a acomodar el aula y luego se iba con una linda sonrisa para su casa. ¡Quién iba a pensar que lo iba a ver en ese momento y hecho todo un hombre! Definitivamente eso vale mucho, son recuerdos que se vienen a la memoria y que tienen un gran significado para las personas; es recobrar la esperanza de que muchos de ellos son personas de una nueva vida, con obligaciones hogareñas, sociales, políticas, económicas y educativas. Conocer qué hacen en la actualidad, lo hace a uno satisfacerse de que son hombres y mujeres de bien, algunos juntados, otros casados y los otros solteros, porque son de la idea de que casarse es un mal negocio, así lo dicen algunos de ellos, quienes han pensado en disfrutar la vida a su manera, para luego pensar en obras más serias, como lo sería un matrimonio.

$\mathrm{Y}$ algo curioso e interesante es que después de haberme ido por más de ocho años de mi escuela para tener una oportunidad de nivel universitario, volví a mi centro educativo donde tengo mi propiedad, y encontrarme en esa zona rural fue 
muy gratificante, como si fuese la primera vez. Muchos de mis alumnos que tuve aproximadamente unos catorce años atrás, llevaban ese primer día de clases a sus niños y niñas, muchos de ellos iban para el nivel de transición, otros para primer grado. ¡Qué grato era verlos como padres y madres de familia, que al verme con gran orgullo les dijeron a sus pequeños que yo había sido su maestra! Incluso preguntaron por qué no les daba primer grado a sus hijos. Pero ese año no era posible, pues iba con gran orgullo a dar un sexto grado, uno de los niveles que me encantan además de primer grado.

Lo que sí es cierto es que han sido personas resilientes y eso les ayuda a sentirse más seguros en su vida, pues esa coraza que lograron construir hizo que muchos de ellos sean hoy en día profesionales de diversa índole.

Pues no sé si a ustedes les pasa, pero cuando tengo tiempo de pensar por dónde he transitado, me llena saber que hemos tocado la vida de muchas personas, son tan excitantes los bellos recuerdos que poseemos y decimos cómo ha pasado el tiempo. En cada recuerdo y acto educativo realizado, se cristalizan nuestras obras como formadores.

¿Por qué hemos perdido el camino en la educación de nuestros niños, niñas, jóvenes y adultos? Hay una infinidad de razones, y algunas hasta la saciedad se han dicho, no obstante, vamos a reflexionar sobre ellas.

\section{Vocación y no ocasión}

Para que tenga sentido lo que hacemos y ello nos provoque un sentimiento de satisfacción, alegría, gozo y placeres, es necesario que usted y yo sintamos que lo que hacemos nos produce felicidad. Por ello, el trabajo no debe ser una tortura ni una cruz, ni una obligación que debe uno cargar por siempre; es, al contrario, la oportunidad de sentir que su labor se ve recompensada por servir a los demás. De ahí que posiblemente muchos de los males que les aquejan a los docentes que están más por la ocasión y no por la vocación se pueden curar. Porque en vez de quejarse de los problemas que les agobian, estarían buscando múltiples alternativas para resolver las situaciones que se presentan en la cotidianidad.

La vocación es un sentimiento de responsabilidad personal, social, profesional que experimentamos muchos educadores cuando transitamos por las aulas, y hacemos que ese espacio se convierta en el acto más emocionante y de aventura para un aprendiente. Y por ocasión es cuando se lleva un proceso de formación condicionado porque posiblemente no hubo alguna otra alternativa para acceder a una carrera universitaria; es ahí donde muchos docentes hacen lo mínimo en el gremio, incidiendo así en los procesos de formación, provocando desgano, apatía, desinterés hacia la enseñanza y aprendizaje por parte de los estudiantes.

\section{Responsabilidad en el acto educativo}

Es posicionarse como protagonistas en el accionar educativo. Es buscar las múltiples posibilidades académicas, pedagógicas 
para ofrecer un proceso de calidad y de entusiasmo en los estudiantes, es convertir a los niños, niñas y jóvenes en líderes sociales para que transiten por la vida y sus contextos de forma significativa. Es, también, evitar que la irresponsabilidad de unos pocos afecte la labor de una colectividad.

\section{¿Qué es la aventura pedagógica?}

Son las múltiples y variadas acciones con que un profesional en educación pone a disposición de sus estudiantes las posibilidades de enamorarse de la autopista del conocimiento, del manejo de la información y de las tecnologías, de la forma de convivir y compartir, de ser cada vez mejor ser humano.

Benjamín Núñez Vargas, primer rector de la Universidad Nacional, decía sobre la impostergable necesidad de atender al joven, de atraerlo a la Universidad Necesaria "más aún, aquel profesional que no se mantenga actualizado en su campo de saber, dejará de ser buen profesional, para convertirse antropológicamente en un fósil viviente" (2008: p.90) y reafirmaba que trabajamos afanosamente para entregar a la sociedad costarricense líderes que encabecen la toma de decisiones, que lleven a cabo innovaciones y sean capaces de construir propuestas para la resolución de los problemas de la realidad nacional.

Es entender que se aprende a ser, a vivir en sociedad para contribuir a la formación de un ser holístico. Es hacer del aprendizaje toda una aventura, única, trascendental, lleno de alegrías, sorpresas, sabores, sinsabores, llantos, desaciertos, sabor de lo aprendido.
Recuerdo a un joven que transitaba por la universidad en su primer año de carrera. Había llegado muy ilusionado, pero una tarde me lo encontré sentado en unas bancas, cuando le pregunté cómo le había ido al finalizar ese periodo, se levantó y me dijo: "Profe, me quedé en una materia, tendré que repetirla el otro año, pero me quedé con gusto y no me arrepiento de lo sucedido".

Al contarme lo que había pasado, me dijo que él se había descuidado al no cumplir con sus responsabilidades como estudiante, pero que las clases de la profesora eran increíbles, llenas de mucho entusiasmo y un conocimiento de la materia que lo dejaba a él complacido, realmente estaba encantado y que todo lo que había aprendido le servía para la vida, es más, él quería que cuando volviese a llevar el curso, que fuese ella la persona que se lo diera, pues se complacería de seguir aprendiendo.

Cuando uno analiza el relato se da cuenta de cómo estos mediadores pedagógicos contribuyen en las acciones que desarrolla el otro/a, y de cómo, incluso, calan en la vida y formación de estas personas; no hay resentimientos y es el mismo aprendiente quien dice que la causa del rendimiento académico es él. Y es que el ser humano necesita estímulos que lo llenen de luz y esperanza para sentir felicidad con lo que hace y cómo lo hace.

Por tanto, esa aventura pedagógica es apasionarnos con todo aquello que la misma naturaleza nos ofrece, que nos hace vibrar, emocionarnos, darnos paz interna, derramar por nuestras venas una espiritualidad celestial de regocijo. Es entrar en empatía 
con todos y el todo, generando así sentimientos positivos y practicar la sanación colectiva, es construir nuestras cotidianidades en aventura y asombros. Es encontrar la salud y la felicidad por siempre.

Cada instante de nuestras vidas debe vivirse placenteramente; estos te marcarán con huellas significativas. Maestros, maestras, padres, madres y comunidades que propicien e irradien sentimientos positivos hacia la vida; es permitirse construir nuevos valores, actitudes y estilos de vida diferente. Necesitamos una cultura naciente, con aulas sin paredes, donde los chamanes, curanderos, cocineras/os, agricultores, y un sinfín de actores propios de los pueblos, enriquezcan los aprendizajes de los aprendientes con la experiencia y sabiduría de sus vidas. Dichos personajes estarán compartiendo el ejemplo de su vida para inspirarnos con ese aliento sagrado: la existencia en todas sus formas.

\section{Principios de la aventura pedagógica}

Hay una constante búsqueda de sentidos y significados para los aprendizajes, por eso, desde una visión holística, se propician los siguientes principios en el entendido que se seguirán construyendo en la convivencia y en las interrelaciones humanas como signos que dan sentido a lo que somos, a lo que hacemos en el hoy y en el mañana. Los sentimientos humanos son el alimento necesario para que los seres humanos tengamos la felicidad y el gozo, porque la vida es para gozarla, disfrutarla, amarla y compartirla, de manera tal que cada uno de estos aspectos enriquezcan las prácticas pedagógicas de las personas, a saber:

\section{Amor}

El amor abre corazones, escucha mutuamente y permite una manera mágica de pensar y sentir. Con el amor emergen sentimientos de armonía, orden interno y serenidad. Permite un estado de plenitud consigo mismo y con los demás. Una educación basada en el amor, la ternura, las emociones en las experiencias de aprendizaje que se propicien permitirá co-crear una cultura solidaria, de respeto y democracia. Esta práctica en instituciones vivas hará que el respeto por sí mismo y por el otro marque las interacciones humanas de socialización. "Si se convive en espacios de amorosidad, nuestro ser amoroso florece, pues todos somos básicamente amorosos e inteligentes" (Maturana, 1999; p.28). El amor es el encanto en acción, lo que crea y recrea vida a otros y luego los invita a unirse a todos.

\section{Solidaridad}

Es la fuente de convivencia social que favorece la armonía, los valores, el conocimiento y las emociones entre los seres humanos para la búsqueda del bien común.

Una educación que forme seres humanos sensibles, solidarios, donde no haya personas opresoras ni oprimidas, donde prevalezca el consejo, la conciliación, la democracia.

Imaginarnos y construir una educación esperanzadora, donde hombres y mujeres puedan hacer más apasionante la vida humana, en todos sus sentidos.

\section{Integralidad}

Es concebida como la acción de las partes con el todo. La persona siente la necesidad 
de conspirar y compartir con otros/as; entrelazar ideas, opiniones, sentimientos de unión, reflexión profunda ante los procesos naturales y sociales.

Fluye la integralidad en lo que Maturana expresa: "hay una red de relaciones dinámicas, en la cual el éxito depende de cada parte y el éxito de cada parte depende del éxito del sistema como un todo". Personas completas e íntegras ante los desafíos de la vida (1999, p.97).

La relación de construcciones juntas y equitativas entre las mujeres y los hombres es una tarea compartida, donde participamos todos/as. Comunica el mensaje de realizar las tareas en equipo, compartir las responsabilidades, buscar la unidad en la diversidad y llenarnos de alegría al trabajar en conjunto. Hace que se promuevan procesos reflexivos hacia la equidad e igualdad entre géneros.

Debemos encaminarnos hacia ese cambio de paradigma. Existe una serie de iniciativas en convenciones, acuerdos, leyes, declaraciones, cartas, que abogan por un mundo mejor, uno que respete, cuide, proteja y preserve la tierra y la vida en su diversidad, cuya sociedad sea democrática, justa, sostenible y pacífica, sin personas pobres ni discriminación por causa alguna, con instituciones que fomenten y propicien la gobernabilidad, la participación inclusiva de la ciudadanía en la toma de decisiones y el acceso a la justicia.

La Carta a la Tierra nos invita a ser parte de esa coalición mundial de personas dispuestas a hacer de esos principios un estilo de vida, que nos permita, como dice el libro de Santiago, dejar de ser solamente oidores/as, para convertirnos en hacedores/as.

En este estadio superior, el consejo de Wangari Maathai Kenya es fundamental: "abandone la apatía y avance hacia la acción”. Según su aporte, debemos tomar cada palabra, permitir que cada una de ellas cobre significado en nosotros/as mediante la pedagogía de la pregunta: ¿Qué es ser justo/a? ¿Cómo puedo manifestar la justicia? ¿Qué hago para cuidar la Tierra? ¿Quiénes están siendo discriminados/as? Y miles de preguntas más que nos hagan reflexionar sobre formas de garantizar la seguridad, sostenibilidad y unión de la vida en la Tierra.

\section{Emociones}

Son los sentimientos más puros y bellos que pueden eclosionar en las personas. Hacen vibrar al ser desde lo más profundo del corazón, para dar paso a sensaciones de placer y gozo por las acciones que hacen. Esa energía positiva que aflora, irradia una luz esperanzadora para el futuro.

Emocionar trae consigo una responsabilidad social corporativa, donde se desperdigan semillas de concienciación inspiradoras hacia el bien común, que se convierte en tejido humano, dispuesto al fortalecimiento del espíritu interior como esperanza de vida, a mantener una relación íntima con la vida misma; a interactuar apasionadamente con las acciones locales colectivas, a desarrollar agricultura sostenible y, principalmente, a fortalecer la solidaridad, la confianza y la cooperación entre las comunidades. Vivimos en lucha 
contra las emociones en el supuesto que ellas nos alejan de la razón.

Las relaciones humanas se ordenan desde la emoción y no desde la razón. Hay momentos especiales reflejados en la memoria de muchos, a partir de sus experiencias. Veamos un ejemplo en un encuentro de socialización ocurrido con docentes de varias direcciones regionales, quienes producto del afloramiento de las emociones y aprendizajes comparten su obra literaria como una forma de expresar sus ideas y sentimientos de lo aprendido.

Dar un paso firme a un cambio de mentalidad y a las formas en que se han dado los procesos educativos, nos hace disconformistas, inquietos, soñadores y divergentes. Nos convierte en protagonistas de un nuevo día, en coreógrafos y no solo espectadores de la vida.

A eso le llamamos el despertar de una nueva conciencia, para enfrentar los retos y desafíos de una sociedad de aprendientes. Así lo reafirma Assman (2002: p.98). Aprender es siempre descubrimiento de lo nuevo; si no, no es aprender. Educar es ir creando continuamente nuevas condiciones iniciales que transforman el espectro de posibilidades de afrontar la realidad.

Este es el punto clave de lo que la pedagogía debería aprender de la teoría del caos. Aprender es un proceso autoorganizativo, en el sentido de creación de lo nuevo. Los procesos emergentes adquieren una dinámica propia en la medida en que van mostrando bifurcaciones. ¿Hacia dónde nos lleva esta nueva concepción de hacer bien las cosas? Hacia procesos educativos de calidad, cautivadores y llenos de emociones.

El acto de educar es toda una aventura; no es solo que los aprehendientes sientan que se despiertan llenos de gozo y placer ante la diversidad de formas en que se educa, sino de procesos de interaprendizaje significativos que construyen. Assman (2002) agrega un enfoque práctico en la orientación pedagógica de cómo podemos recuperar las emociones en todo momento de la vida. Indica que el juego pedagógico da lugar a lo lúdico, a la alegría de construir, abre espacios a la creatividad y al interaprendizaje. Los ejercicios prácticos de significación, expresión, resignificación y recreación, autopercepción e intertextualidad provocan escenarios diversos de autoaprendizaje. En esta misma línea, Maturana (1999) aporta que el aprendizaje significativo debe tener una dosis de gozo, creatividad, placer, incertidumbre; con ese proceso creativo el ser vivo se autoorganiza y se autoconstruye.

El cerebro es un auténtico órgano social, necesitado de estímulos ambientales para su desarrollo. Sin apego afectivo, no puede alcanzar sus cumbres más elevadas en la aventura del conocimiento.

Desde este punto de vista, no se deben dejar de lado dos aspectos importantes que inciden en el aprendizaje: el dominio racional y el dominio emocional, poniendo especial énfasis en las emociones por medio del amor. El amor constituye una emoción, que se siente, se vive y es el modo de vivir juntos, el cual permite que aflore una serie de vivencias significativas. 
Maturana (1999) enfatiza en que debemos reconocer el amor como la emoción que justifica lo esencial, con valores bien fundados, como la honestidad, solidaridad, respeto mutuo, colaboración, equidad. Esto da cabida a sentimientos de satisfacción y gozo, desde la vida cotidiana.

\section{Disfrute intelectual}

Es la capacidad que tienen las personas para gozar plenamente de las experiencias de aprendizaje que entran por los sentidos. Conseguir armonía entre el sentimiento y el placer de descubrir es provocar "sabor al saber”, desencadenándose así vivencias sinérgicas que transformarán sus conocimientos. La satisfacción, complacencia y provocación de nuevos aprendizajes conducirán al disfrute pleno de todas las capacidades del ser humano. Gallegos (1997) manifiesta que "el conocimiento surge solo si es animado por la inteligencia, el discernimiento para reconocer y dar sentido".

Facilitar el conocimiento es desencadenar aprendizajes, dar pistas de procesos agradables, entusiastas y motivadores. Se debe dejar constancia del autoaprendizaje en todas las manifestaciones de nuestra vida.

\section{Diálogo}

Son espacios de comunicación donde se conjugan los sentimientos, inquietudes, opiniones, emociones, para la toma de decisiones. Entran en juego las diferentes formas verbales que promueven relaciones y los aprendizajes. En estos espacios se expresa con el cuerpo y mente, a fin de que aflore la conciencia reflexiva y transformadora de realidades.
Son espacios abiertos, donde fluye el lenguaje, con personas dispuestas a conversar. Es un espacio de acciones, respeto mutuo, donde convergen posiciones diversas y toma de decisiones. Humberto Maturana manifiesta que sin el placer de la compañía, sin amor, no hay socialización humana y toda sociedad en la que se pierde el amor, las interacciones se pierden. Ese diálogo interno y externo le da sentido a las cosas, abriendo así el espacio para la pedagogía de la pregunta y la pedagogía de la esperanza.

Entra en juego un currículo visto como un conjunto de relaciones, con especial énfasis en el lenguaje, imaginación y análisis como aspectos importantes del conocimiento y las habilidades comunicativas.

Conscientes de los intercambios y reflexiones en torno de la construcción de una nueva educación, es posible esbozar una experiencia con un grupo de docentes de diversos contextos educativos, quienes fueron testigos cómplices para aventurarse a ser gestores de cambio en sus comunidades rurales.

Estas fueron algunas de las expresiones dadas en un taller de seducción y sensibilización que se les brindó, como parte de los cambios paradigmáticos que se pueden producir, si la persona es entusiasta, con capacidad de enfrentar los desafíos de lo cotidiano. $\mathrm{Y}$ es que el entusiasmo es un estado de fe, de afirmación de sí mismo, con una fuerza interior de transformar el mundo, movidos por la fuerza y la certeza de nuestras acciones.

Estos esfuerzos evidencian que hay esperanza en otro amanecer en las comunidades rurales. Coincidimos en que debe 
existir un cambio de actitudes en las personas que educan, para la construcción de comunidades de aprendizajes que posibiliten la generación de la nueva realidad. Es una oportunidad para enriquecernos como profesionales, como personas que tenemos en nuestras manos seres tan importantes como los niños/as.

En definitiva, el lenguaje es una fuente de inspiración donde se estimula la significación y la razón. Dialogar llena los vacíos de las preguntas. Necesitamos un nuevo tipo de diálogo, que permita escucharnos mutuamente, para estrechar nuestras relaciones.

El bienestar total, tanto físico, psicológico y espiritual, es un complemento esencial en los seres humanos. Esa energía vital que cada uno irradia es necesaria para prepararse y enfrentar cualquier situación de incertidumbre. Tener en los ambientes educativos a un grupo de aprendientes con características y cualidades propias, con armonías de vida diferentes, es todo un desafío. Razón por la cual los mediadores deben diagnosticar los perfiles de los jóvenes que tienen bajo su responsabilidad; ellos necesitan enseñar nuevos mitos, epopeyas, cuentos en los cuales los seres humanos son bondadosos, mujeres y hombres pacíficos y el poder de la creatividad y el amor, simbolizado por el cáliz sagrado, es el principio supremo de la transformación de la sociedad.

\section{Convivir}

Proceso de educar y educarnos en las relaciones cotidianas, niveles de convivencia donde aflore el afecto, la socialización, los sentimientos y la ayuda recíproca entre los seres. Seres humanos capaces de realizar actos conscientes, socialmente responsables. Se debe generar un ambiente de cohesión social, donde la relación dialógica estimule el sentido de la vida misma, en armonía, paz y felicidad.

Crear una visión holística del planeta permite al ser humano integrar sus ideas, conocimientos al servicio de la humanidad y del mundo. Por lo que los ambientes pedagógicos que se estimulen deben estar orientados a experiencias directas desde sus cotidianidades.

\section{Propiciando un nuevo lenguaje}

La mediación pedagógica busca que los procesos tengan aprendizaje con experiencias significativas, llenas de gozo, placer, novedosas; por ello, la expresividad es un talento que debe desarrollarse mediante espacios donde se opine, discuta, critique con claridad, con fundamento, coherencia, seguridad.

Hay que promoverla en sus tres dimensiones: verbal, oral y escrita. Respetar las opiniones e ideas ajenas es permitir que cada persona se sienta protagonista. Estas experiencias deben lograr la interlocución y la interactividad entre los aprendientes.

Propiciar el emocionar como un modo inconsciente de ver, oír, reflexionar, comprender, aceptar, razonar y hacer.

El ambiente pedagógico tiene que ser un lugar de fascinación e inventiva: no inhibir, sino propiciar la dosis de ilusión común entusiasta requerida para que el proceso de aprender se produzca como mezcla de todos los sentidos con los que 
captamos corporalmente el mundo. Todo conocimiento tiene una inscripción corporal, y que venga acompañada de una sensación de placer no es, en modo alguno, un aspecto secundario.

\section{Inspiraciones desde la perspectiva de Joaquín García Monge}

\section{Inspirar para la fascinación}

Muchos autores en el área de la pedagogía agregan que si empleamos música, cuentos, juegos, proyectos para enseñar respeto mutuo y ayudar a los niños (as) y jóvenes un sentido de comunidad, estamos contribuyendo a ejercitar sus mentes y corazones, más sensibilizados, ante las personas y el entorno que les rodea. Así lo esbozaba el gran maestro García Monge al indicar que se recomiendan las investigaciones personales de los alumnos como un medio de darle importancia a las asignaturas. Interesar a los alumnos en las ciencias naturales. Se piden conferencias científicas de los alumnos para sus compañeros. Se recomienda a los jóvenes las investigaciones originales como las mejores fuentes de estudio. (Rodríguez Vega, 2001)

Incluir, en los procesos educativos, la novedad, la creatividad y la imaginación despertará en ellos (as) los niveles emocional, espiritual, físico, artístico, cognitivo $\mathrm{y}$ espacial de manera interrelacionada. Esta visión holística facilita una percepción integral de la realidad, formando seres humanos conscientes de su entorno.

Morín (2001) propone en su libro Los siete saberes necesarios, las ecologías de la acción por medio de las incertidumbres, como una oportunidad de explorar el conocimiento como aventura incierta ante el riesgo de la ilusión y el error.

\section{Inspirar para redescubrir con pasión}

Las personas que buscan lo que les apasiona, contribuyen a la unión de todo el universo; se dice que la unidad del mundo depende de la búsqueda de lo que nos apasiona. La vida surge en respuesta al encanto que poseemos internamente y deseamos exteriorizarlo hacia otros; esta secuencia revela el sentido del esfuerzo humano capaz de desarrollar sus potencialidades, dando paso a la imaginación y la creación. Es en la naturaleza donde los seres humanos podemos imaginarnos formas de buscar la sostenibilidad, solidaridad, políticas activas de inclusión, espíritu emprendedor, entre otras capacidades.

Si ejercitamos estas acciones, los aprendientes tendrán una visión más holística de la vida en cotidianidad. Es necesario enseñar a mostrar cómo mediante las observaciones hay situaciones maravillosas que pueden salir de ellas. Por ello, inspirarnos con el aliento sagrado de la existencia en todas sus formas es saborear la vida cotidiana con alegría profunda, perdurable y con placer; son el alimento de la autosanación y la sanación colectiva.

La pasión por aprender es un ingrediente indispensable en la maravillosa tarea de educar; por ello, coincide en que los mediadores deben viajar junto con su aprendiente en la maravillosa tarea de educar, en un mundo de aventuras para descubrir las maravillas que hay en él. Así mismo, 
dice: “...quizás un maestro solo sea una persona que facilita, que coloca cosas delante de la gente y muestra cuán emocionantes y maravillosas son, incitando a probarlas". Enseñar lo mágico, lo absurdo, lo misterioso es hacer viajes insospechados por los caminos del aprendizaje. Descubrir y descubrirse a uno mismo permite el deleite y la transformación con la plena seguridad de que nuestra conciencia se libera y nuestra mente se abre a un mundo de posibilidades.

\section{Inspirar para fortalecer la convivencia}

Dar y compartir el aliento de la vida es conectarnos con las personas. Si cada uno de nosotros se levanta cada mañana, descubre la alegría de la vida diaria, derrama optimismo, esperanza, muchos otros recibirán esta respuesta para, de igual forma, aprender a celebrar la comprensión del significado de la vida.

Dossey manifiesta en su libro El poder curativo de la mente (2004) que estamos sedientos de un sentido y eso hace que el mundo cotidiano de muchas personas carezca de encanto. Por tanto, si buscamos nuestro interior y lo cargamos de mucha paz, amor, espiritualidad, esto generará sentimientos positivos para un enfoque espiritual de la vida; esto permite ir más allá, crea una resonancia afectiva en los demás seres humanos.

En los hogares, la capacidad de amar y de sentirnos amados motivará a dar en el futuro la medicina espiritual en espacios de convivencia, que tanta falta hacen. Los espacios de convivencia diaria no son nada fáciles, exigen esfuerzo de aceptar al otro, tal y como es, a respetarlo y a crear un nivel de tolerancia alto. Así mismo, con la convicción de que podemos ayudarle a cambiar, si fuese necesario.

Convivir es compartir amor, temores, angustia, crisis, caos, incertidumbre... tomar parte en una vida ajena, muchas veces totalmente diferente de la de uno y hacer partícipe de la propia. Transformar el vivir juntos es enseñar a vivir en las interacciones, en un mundo donde estamos interconectados con los fenómenos naturales. La complementariedad en los seres humanos es una forma de validar la convivencia. Cuando tenemos la oportunidad de convivir juntos, disfrutamos ese espacio para crecer y transformarnos.

\section{Inspirar para la autogestión}

Proceso que se construye desde adentro y desde abajo, donde las personas manifiestan sus sentires y desarrollan sus capacidades endógenas. Las poblaciones pueden expresarse, ser escuchadas y con sus capacidades críticas y propositivas plantear propuestas.

Las comunidades tienen sus realidades y los estudiantes, siendo líderes sociales, pueden retomar estos escenarios para resolver los problemas que les aquejan, toman decisiones, con el fin de asegurar:

- Valores humanistas y ambientales

- Nueva economía para la conciencia ambiental

- Nuevo orden social

- Armonía ambiental 
- Crear nuevas formas de ser y de estar en este mundo

- Revolución espiritual

\section{Inspirar hacia una nueva conciencia social}

Los espacios de convivencia que se generan permiten compartir sueños, ilusiones, iniciativas con nuevos lenguajes y formas de actuar. Convivir es compartir el esfuerzo diario, fortalecer las relaciones, el respeto. La vida humana ha de existir en un orden, unas conexiones y secuencias sucesivas.

Es la oportunidad de retomar el "Grito de Vargas "y "el Grito de Tillarán”, como voces conspiradoras, llenas de esperanzas, que nos instan a retomar el camino hacia la construcción de una sociedad solidaria, donde las personas valoren otras formas de percepción y conocimiento de sus propias realidades.

En este contexto, es necesario que la educación recupere su finalidad humana, que por medio del conocimiento podamos diseñar nuevas instituciones, las cuales permitan la subsistencia del ser humano planetario del siglo XXI.

\section{Algunas ideas presentes en los educadores y pensadores de Repertorio Americano}

A- Expresar y pronunciar un nuevo modo de sentir la realidad, buscando el equilibrio dinámico de los seres, excitados por descubrir, crear, soñar, conspirar, con la apertura de un nuevo camino. Aulas abiertas, en contacto directo con la realidad... se aprende desde ellas.
B-. Comprender y analizar el verdadero sentido de la formación de los seres humanos, que favorezca la toma de decisiones acertadas hacia una sociedad costarricense más digna, justa y humana.

C-. Generar respuestas eficaces y eficientes a las necesidades educativas de las zonas rurales de Guanacaste, con la inserción de los estudiantes en proyectos sociales y, de esta manera, contribuir con el mejoramiento de la calidad de los procesos educativos en el contexto rural.

D-. Penetrar en los corazones de los aprendientes para la búsqueda de alternativas pedagógicas desde la territorialidad, la sostenibilidad, la recursividad y la creatividad de todos/as los/as jóvenes desafiantes que quieran re-encantar la educación desde sus nichos de aprendizajes.

E-. Revisar y releer el pasado para visualizar y rediseñar el presente y el futuro. En esta era no podemos estar a merced de las fuerzas ciegas, es posible imaginar y soñar un futuro prometedor para la región, tomando como punto de partida la cotidianidad, su propia realidad: su historia, costumbres, tradiciones, leyendas como parte del currículo de hechos, acciones y fenómenos.

Hay una idea de García Monge que confirma la importancia de darle mayor sentido a las prácticas pedagógicas desde el escenario natural por excelencia, la madre naturaleza. Manifiesta que: "La enseñanza en medio de la naturaleza, fuera de las aulas, responde a la necesidad fundamental de hacerla más práctica, más penetrante y de más vastos resultados. Aquellas 
virtudes que en el aula se adormecen y acaban por extinguirse, surgen vigorosas en frente de la naturaleza. La sustitución del material que ella nos ofrece, por otro artificial, desplazado del lugar propio, no es de ninguna manera el mejor procedimiento para desenvolver las capacidades de observación, de comprensión y de juicio" (en Rodríguez, 2001).

Para lograr este cometido, las instituciones tienen la palabra. Y es por medio de sus mediadores/as dispuestos/as a provocar asombro, placer y cambio social. Se logra con el desarrollo de clases creativas, construyendo, creando y recreando conocimientos, con formas de convivencia donde el amor, el respeto, la solidaridad, la igualdad y la equidad constituyen alicientes para la cocreación de sociedades solidarias.

Supone la participación de personas indagadoras de lo nuevo, sistemáticas en la búsqueda de antecedentes, experimentadoras, que ponen a prueba lo aprendido, inconformistas con lo que aprenden, inquietas para saber más, soñadoras y divergentes. Es una propuesta de cambio de mentalidad y, consecuentemente, de las relaciones sociales y las formas de organización existentes.

"Nuestra escuela -escribe García Monge en 1920 - ya tiene algunas conquistas liberales y democráticas a que otros pueblos mayores aspiran: el laicismo y la coeducación, por ejemplo, las actividades sociales y económicas, ciertas actividades físicas y educacionales como los trabajos manuales, la agricultura y la cocina, todo amenazado ahora por la incomprensión y las antipatías de numerosos ciudadanos y ciudadanas. La resolución de los maestros y de los Patronatos salvará estas cosas" (en Rodríguez, 2001).

Este pensamiento producto de la praxis es la oportunidad de compartirlo; a lo mejor hay tantas experiencias bellas que están ahí en la mente de muchos educadores que solo les hace falta sentarse muchos minutos para dejarlas impresas en la historia. Recordando al maestro García Monge, en una de las páginas de sus escritos decía: "Gritos de vida vibrarán en las páginas de esta revista, gritos vivientes y soberbios que harán despertar a los dormidos en el fondo de las aguas estancadas de nuestro mundo moral e intelectual" (1 de abril de 1904), citado por Rodríguez (2001).

\section{Conclusiones para el disfrute de retomar las acciones del pedagogo, inspiradas en el Repertorio Americano}

Brindar capacitación permanente a los mediadores educativos para la transformación de pensamientos, percepciones y valores; dialogar, interactuar con otros/as nos permite abrirnos hacia el nuevo paradigma. Mediante el intercambio y entretejido de informaciones, textos y experiencias, se va construyendo y dando sentido a las intencionalidades individuales y grupales.

Buscar seleccionar estrategias y técnicas que den sentido y significado a los espacios educativos que se les proporcionan a los aprendientes; por ello, las siguientes recomendaciones podrán ser útiles en la medida en que se pongan en práctica, se logre el objetivo a plenitud e, incluso, más allá de lo planeado: 
Realizar encuentros de socialización en zonas llenas de belleza natural, que contagien e inspiren nuevos aprendizajes desde la vida misma. Es alfabetizarnos ecológicamente en espacios seductores, donde cada aprendiente se nutrirá con un nuevo diálogo con la naturaleza, tal y como lo plantea Frijot Capra: “Aprender (de ella) de su complejidad y belleza mediante el respeto, la cooperación y el diálogo".

Excursiones, giras académicas, observar y disfrutar ambientes ricos en vegetación es una motivación para reconquistarnos con la trama de la vida. Recuperar este derecho es lo que Michio Kaku dice que estamos en la cúspide de una transición: de ser observadores pasivos de la naturaleza, a ser coreógrafos activos de la naturaleza. Si permitimos hacer de esta época la más apasionante, con sentimientos profundos de hacer, conservar, estaremos recogiendo los frutos en una época no muy lejana.

Aplicar técnicas bioenergéticas como el bio-feedback, entrenamiento autógeno, meditación trascendental, de relajamiento progresivo, ayuda a los niños/as y jóvenes para dejar de lado las tensiones, angustias, temores que traen de sus hogares.

Promover proyectos educativos comunales: acciones verdes. Cada una de las problemáticas será abordada por prioridad; ellos y ellas buscarán alternativas viables de ejecutar, es aprender mostrando. Ello seduce la imaginación, los entusiasma a la toma de decisiones y a la creación de proyectos ecológicos, de cuido y preservación de sus territorialidades.
Propiciar espacios constantes de reflexión, como fuente de discusión para los temas de interés, liderados por los mismos aprendientes. Surge, sin lugar a dudas, la creatividad, la criticidad, el diálogo y la armonía. Niños/as, jóvenes, adultos, creando espacios de diálogo, permitirán cambiar el mundo en que vivimos. Maturana, en su libro: El sentido de lo humano, nos hace reflexionar cuando dice: "que, si nos sumergimos en la búsqueda de las apariencias y en las jerarquías que niegan la solidaridad y la reflexión, desvirtuamos lo fundamental de nuestro ser humano.

Generar un modelo solidario como vía de crecimiento personal y espiritual, incorporar a todos los actores sociales. Es un tejido social donde prima la solidaridad, el compañerismo en búsqueda de la espiritualidad solidaria.

Con nuestros aprendientes se podrán llevar a cabo, mediante talleres de autoconocimiento, relaciones humanas, talento humano, clubes de tertulias, conversatorios, caminatas amigables, tienda de valores, visitas a centros de asistencia social: hogar de ancianos, hogar de niños y un sinfín de ideas que pueden originarse para la práctica de valores de convivencia. Incluso, incorporar a padres y madres en proyectos colaborativos que seduzcan a otras personas a unirse y formar una red social.

Una propuesta de asociatividad, bajo las modalidades de cooperativas escolares, organizaciones de hombres, mujeres o grupos mixtos, que se logren incentivar en escuelas rurales, no solo permitirá las verdades repúblicas ciudadanas, sino el fortalecimiento de las comunidades con formas 
de producción autogestionarias, lo mismo que el poder organizado en lo económico, político y social de los aprendientes.

En este sentido, tanto la niñez, los jóvenes, los padres y madres de familia y comunidad en general, pueden constituirse en cooperativas de autogestión, de producción, cuyos principios estarán sustentados en la libertad, participación voluntaria, la autosustentabilidad económica que les presenta su adscripción como asociados/as a empresas solidarias.

La característica de la educación ofrecida en el medio rural debe tener la intencionalidad de vincular a la niñez, jóvenes, padres y madres de familia y comunidad en general, en verdaderas comunidades de aprendizaje, donde el contexto rural genere oportunidades de desarrollo económico y social. La educación agrícola ha sido el vehículo en educación primaria para integrar a todos los actores sociales, por la naturaleza que representa, ha estado llamada a crear una identidad campesina; y esta ha sido una forma importante de acercar la escuela con la comunidad y de ser docentes generadores de cambio.

Practicar un lenguaje inclusivo en todas sus manifestaciones, para que se valore la diversidad de género, raza, religión o etnia. Desmitificar todo aquello que durante mucho tiempo nos ha atado a mitos y realidades dolorosas e insanas. En canciones, poemas, lecturas, cuentos; en todo tipo de lectura donde se hace evidente a las personas adaptarse a rangos de dominado-dominador.
Continúa diciendo la misma autora que debemos evitar el servilismo, sufrimiento, humillación, por bendiciones, alegrías, padres y madres amorosos que obtienen placer de nuestra felicidad, un balance entre necesidades tanto físicas como espirituales. ¡Busquemos valores y lenguaje inclusivo en nuestras prácticas pedagógicas!

Estimular la creación de comunidades de aprendizaje, para que juntos luchen en la conformación de proyectos comunitarios que incluyan a actores sociales: maestros/as, niños/as, padres, madres de familia, instituciones organizadas como organismos vivos. Es una forma de mirar el mundo desde la territorialidad. Fomentar el sentido de comunidad dentro del aula y fuera de ella, basado en el respeto mutuo, normas establecidas en conjunto con los aprendientes, crear un ambiente colaborativo, hacer magia, impregnar el conocimiento desde diferentes formas de conectar el aprendizaje.

Fortalecer el sistema de valores, sustentándolos con el medio natural, social, es sensibilizarnos de la riqueza de la vida que está a nuestro alrededor, que desde lo más profundo de nuestro ser encontremos sentido de nosotros mismos, con las otras personas y con lo que nos rodea. Convivir para vivir es la posibilidad que tenemos los seres humanos para regenerarnos permanentemente desde lo intelectual, ético, psíquico, moral y tecnológico.

Generar comportamiento ecológico en todas las acciones humanas, mediante una vida llena de solidaridad, disciplina, conciencia planetaria y es aquí donde la mediación pedagógica cobra sentido, por 
medio de las experiencias que se susciten, las cuales deben basarse en despertar interés, satisfacción, creatividad, libertad, pero principalmente, ayudar a transformar la realidad. Incorporar el trabajo colaborativo, utilizando las tecnologías de la información y comunicación para desarrollar aprendizajes significativos.

Construir en los centros educativos y en las comunidades espacios de libertad, donde el ser humano tiene la posibilidad de hacer sus propias elecciones y toma de decisiones, bajo un ambiente de confianza, esperanza, perseverancia de los sueños e ilusiones compartidas.

\section{Bibliografía}

Assmann, H. (2002). Placer y ternura en la educación: hacia una sociedad aprendiente. Madrid, España: Nancea S.A de Ediciones.

Boff, L. (2002). Ecología: grito de la Tierra, grito de los pobres. Madrid, España: Editorial Trotta.

García Monge, J. (1920). Memoria de Instrucción Pública. En Garrón de Doryan, V., p. 70. (27). Memoria de Instrucción Pública.

García Monge, J. (1923). “A propósito del 10 de mayo", citado por Garrón de Doryan, V. en Repertorio Americano, 30 de abril 1923. No. 3, pp. 101-109.

García Monge, J. (1974). Obras escogidas de Joaquín García Monge. San José, Costa Rica: EDUCA.

Capra, F. (2003). Las conexiones ocultas. Barcelona: Editorial Anagrama.

Capra, F. (1996). La trama de la vida. Barcelona: Editorial Anagrama.
Capra, F. (1982). El Punto Crucial. Argentina: Editorial Troquel.

Cosachov, M. (2000). Entre el cielo y la tierra. Un viaje por el mapa del conocimiento. Buenos Aires, Argentina: Editorial Biblos.

Dossey, L. (2004). El poder curativo de la mente. La salud más allá del cuerpo. México: Santillana.

Gallegos, R. y otros (1997). El destino invisible de la educación. México: Editorial Pax.

Gutiérrez, F. y Prieto, D. (1994). La mediación pedagógica para la educación popular. San José, Costa Rica: Radio Netherland Training Center.

Maturana, H. (1999). Transformación en la convivencia. Santiago, Chile: Dolmen.

Maturana H. (1995). Emociones y lenguaje en educación y política. Santiago, Chile: Dolmen.

Mora, H. y Hinkelammert, F. (2003). Por una economía orientada hacia la vida. Revista Economía y sociedad. Escuela de Economía de la Universidad Nacional. No. 22.

Morín, E. (2003). El método. La humanidad de la humanidad. La identidad humana. Madrid, España: Ediciones Cátedra. Colección Teorema.

Morín, E. (2001). Los siete saberes necesarios para la educación del futuro. Barcelona, España: Ediciones Paidós.

Payán, J. C. (2000). Lánzate al vacío. Bogotá, Colombia: McGraw-Hill Interamericana.

Rodríguez Delgado, R. (1997). Del universo al ser humano. España: McGraw-Hill.

Rodríguez Vega, E. (2001). Cinco educadores en la historia. San José, Costa Rica: EUNED. 
Soto, J. (2009). Joaquín García Monge:

La Educación Cívico- Social del ciudadano (citado por. • op. cit. • pp 85-88. Cfr. Brenes Mesén, R y García Monge, J. Proyectos de Programas de Instrucción Primaria, Tip. Nacional, 1908, p. XY: "La enseñanza con estudio previo por parte del maestro, para que no divague ni derroche su tiempo" Recuperado de http://www.inif.ucr.ac.cr/ recursos/docs/Revista\%20de\%20 Filos of $\%$ C $3 \%$ AD a $\% 20 \mathrm{UCR} /$ Vol.\%20XXV/No.\%2061/Joaquin $\% 20$ Garcia\%20Monge. $\% 20$ La\%20educaci\%C3\%B3n\%20civico-social\%20del\%20ciudadano. pdf.( Consulta 26 agosto 2016 ) 